\title{
Sustentabilidad de los agroecosistemas de la microcuenca Paso de Ovejas I, Veracruz, México*
}

Bernardino Candelaria-Martínez ${ }^{* *}$, Octavio Ruiz-Rosado***, Ponciano Pérez-Hernández**** Felipe Gallardo-López ${ }^{* * * * *}$, Luis Vargas-Villamil******, Ángel Martínez-Becerra*******, Carolina Flota-Bañuelos $* * * * * * * *$

Recibido: 2013-03-20 Aprobado: 2013-10-I2 Disponible en línea: 2014-05-30

doi:I0.III44/Javeriana.CDRII-73.sdam

Cómo citar este artículo: Candelaria-Martínez, B., Ruiz-Rosado, O., Pérez-Hernández, P., Gallardo-López, F., VargasVillamil, L., Martínez-Becerra, A. y Flota-Bañuelos, C. (20I4). Sustentabilidad de los agroecosistemas de la microcuenca Paso de Ovejas I, Veracruz, México. Cuadernos de desarrollo rural, II(73), 87-104. doi:I0.III44/Javeriana.CDRII-73.sdam

\section{Resumen}

En este texto se planteó como objetivo desarrollar un índice agregado para evaluar la sustentabilidad en los tres grupos de agroecosistemas presentes en la microcuenca Paso de Ovejas i y se planteó como hipótesis que los sistemas con presencia del componente ganadero presentarían un mayor grado de sustentabilidad, debido a un uso complementario de los recursos. Para el presente estudio se definió la sustentabilidad agrícola como el proceso de obtención de productos agropecuarios que satisfagan necesidades de la población, mediante un conjunto de prácticas agrícolas que logran conservar y mejorar la cantidad y calidad de los recursos naturales, y de este modo asegurar su permanencia y mejorar el desempeño de los AES en el tiempo.

\section{Palabras clave autores:}

desarrollo rural; agroecología tropical; ecoganadería

\section{Palabras clave descriptores:}

desarrollo rural; ecología agrícola

* Este artículo se realizó en el marco de la investigación Rediseño participativo de agroecosistemas sustentables en la microcuenca Paso de Ovejas I, Veracruz México, con financiamiento de la línea de investigación en Agroecosistemas Sustentables del Colegio de Postgraduados. El trabajo de campo se realizó de agosto de 2008 a febrero de 201 .

**Doctor en Agroecosistemas Tropicales. Investigador del Instituto Tecnológico de Tizimín. Correo electrónico:bcm8003@gmail.com

*** Doctor en Agroecología. Investigador del Colegio de Postgraduados, campus Veracruz. Correo electrónico: octavior@colpos.mx

****Doctor en Ganadería. Investigador del Colegio de Postgraduados, campus Campeche. Correo electrónico: pperez@colpos.mx

******Doctor en Agroecosistemas Tropicales. Investigador del Colegio de Postgraduados, campus Veracruz. Correo electrónico: glfelipe@colpos.mx

${ }^{* * * * * *}$ Doctor en Ciencias Agropecuarias. Investigador del Colegio de Postgraduados, campus Tabasco.

Correo electrónico: lvillamil@colpos.mx

*******Doctor en Agricultura. Investigador del Colegio de Postgraduados, campus Tabasco. Correo electrónico: mbangel@colpos.mx

********* Doctora en Agroecosistemas Tropicales. Investigadora del Instituto Tecnológico de Tizimín. Correo electrónico: cflotab@gmail.com 


\title{
Agroecosystems Sustainability of the Micro-watershed Paso de Ovejas I, Veracruz, Mexico
}

\begin{abstract}
In this text it is set as a goal the development of an aggregate index for assessing sustainability in the three agroecosystem groups present in the watershed Paso de Ovejas I and it was hypothesized that the systems with presence of stockbreeding would have a greater sustainability due to a complementary use of the resources. For the present study, agricultural sustainability was defined as the process of getting agricultural products that meet the population needs through a set of agricultural practices that conserve and improve the quantity and quality of natural resources, and thus ensure their permanence and enhance the performance of the AES over time.
\end{abstract}

Keywords authors:

rural development; tropical agroecology; ecological stockbreeding

Keywords plus:

rural development; agricultural ecology

\section{Durabilité des agro-écosystèmes du micro-bassin Paso Ovejas I, Veracruz, Mexique}

\section{Résumé}

Dans ce texte, le but proposé cherche le développement d'un indice global pour l'évaluation de la durabilité dans les trois (3) groupes d'agro-écosystèmes présentes au micro-bassin Paso de Ovejas I et l'hypothèse proposée expose que les systèmes qui ont la présence du composant d'élevage de bétail présenteraient un plus grand dégrée de durabilité grâce à une utilisation complémentaire des ressources. Dans cet étude, la durabilité agricole a été définie comme le processus d'obtention de produits agricoles qui parviennent à satisfaire les nécessités de la population, à travers d'un ensemble de pratiques agricoles qui réussissent à préserver et à améliorer la quantité et la qualité des ressources naturels et, de cette manière, à garantir son permanence et l'amélioration de la performance des AES au fur et à mesure du passage du temps.

\section{Mots-clés auteurs:}

développement rural; agro-écologie tropicale; élevage écologique de bétail

\section{Mots-clés descripteur:}

développement rural; écologie agricole 


\section{Introducción}

El desarrollo sustentable es en la actualidad uno de los temas de mayor importancia en las agendas políticas globales y programas de diferentes centros de investigación (Bartlett, 2006; Colás, 200I). En términos generales, se ha propuesto que las metas finales de la sustentabilidad sean autonomía, oportunidades, seguridad y salud de los individuos (McMichael, Butler y Folke, 2003), con todas sus implicaciones en cuanto a los aspectos ambientales, sociales, económicos y políticos. Dentro de la noción global de desarrollo sustentable, la agricultura es concebida como una de las actividades más importantes que debe atenderse, dada su contundente influencia en el desarrollo humano, social, económico, así como en la calidad de los recursos naturales. En México se reporta que 60\% de las localidades rurales con menos de 2500 habitantes presenta un nivel de alta y muy alta marginalidad (Consejo Nacional de Evaluación de la Política de Desarrollo Social [Coneval], 2007), y como país ocupa el lugar número 40 de 45, en el subíndice Manejo Sustentable del Medio Ambiente (Instituto Mexicano para la Competitividad [IMCO], 2007).

Por su parte, Vant, Bakken, Bleken, Baadshaug, et ál. (2006) mencionan que la agricultura moderna puede considerarse como altamente productiva, pero sus efectos negativos en el medio ambiente se han incrementado de forma visible. En esto coincidieron Flota-Bañuelos, Lopéz-Collado, Vargas-Mendoza, Fajersson, GonzálezHernández y Martínez-Morales (20I2), cuando evaluaron escarabajos estercoleros como bioindicadores de perturbación en sistemas ganaderos. En este sentido, Altieri y Nicholls (2012) mencionan que es parte de un modelo de agricultura que se instauró bajo el supuesto de que siempre habría agua en abundancia, energía barata y que el clima no cambiaría.

En las últimas tres décadas los investigadores se han ocupado de construir conceptos de sustentabilidad agrícola, desarrollar teorías, métodos e instrumentos para evaluarla. En la última década se propusieron diferentes marcos para evaluar la sustentabilidad agrícola, desde la perspectiva ambiental (Belcher, Boehm y Fulton, 2004; Torres, Rodríguez y Sánchez, 2004), económica y social (Sepúlveda, Chavarría, Rojas, Castro et ál., 2005), multidisciplinaria (Nahed, Castel, Mena y Caravaca, 2006; Urzelai, Olazábal, García, Santa Coloma, Herranz, Abajo et ál., 2006; Masera, Astier y López, 1999).

Sin embargo, la mayoría de estos marcos toma como referencia información generada en contextos externos y, en ocasiones, con diferentes características. De ahí la necesidad de referenciar la comparación con datos regionales, generados incluso en los propios sistemas, con el fin de evaluar avances y retrocesos, en un escenario justo y acorde a las posibilidades de desarrollo de la microcuenca. 
Por otra parte, Ruiz (200I) destaca la necesidad de adoptar una visión agroecológica que resalte la interacción entre los elementos del agroecosistema con los elementos externos. Asimismo, Cáceres (2004) plantea la necesidad de establecer parámetros contextuales en la definición de sustentabilidad, y concuerda con Nahed et ál. (2006) sobre la importancia de analizar de forma integral la sustentabilidad, y expresarla en un índice agregado. Este, sin embargo, debe permitir evaluar por aparte cada una de las dimensiones e indicadores para detectar puntos clave en el diseño de estrategias ad hoc en los sistemas agrícolas (Candelaria, 20II), dado que en estos pueden estarse desarrollando procesos que comprometen la sustentabilidad a mediano o largo plazo (Cáceres, 2009); esta perspectiva permite, además, ayudar a comprender estos mismos procesos para desarrollar escenarios mediante modelos de simulación, con gran utilidad para lograr el equilibrio en el manejo de los recursos naturales, humanos y económicos (Candelaria, Ruiz, Gallardo, Pérez, Martínez y Vargas, 20II).

En México, el Fideicomiso de Riesgo Compartido (Firco, 2005a) propuso la microcuenca Paso de Ovejas como un espacio idóneo para la atención integral. En el municipio que lleva el mismo nombre, en Veracruz, existen dos microcuencas (Firco, 2005b), Paso de Ovejas I (MPOV-I) y Paso de Ovejas 2 (MPOV-2). La primera es la de mayor vulnerabilidad debido a que se encuentra en la parte de lomeríos, con presencia de agricultura campesina de subsistencia.

En esta microcuenca se han reportado tres grupos diferentes de agroecosistemas (AES), a saber: $\mathrm{G}_{\mathrm{I}}$ ) con tendencia a la ganadería, alta disponibilidad de tierras agrícolas ( $\bar{X}=31$ ha) con número intermedio de prácticas agrícolas ( $\bar{X}=7$ ha); $\mathrm{G}_{2}$ ) con tendencia al manejo de cultivos, baja disponibilidad de tierras $(\bar{X}=7 \mathrm{ha})$, menor cantidad de prácticas agrícolas ( $\bar{X}=4.8$ ha); y $\left.G_{3}\right)$ con tendencia a la ganadería, disponibilidad intermedia de tierras ( $\mathrm{X}=\mathrm{I} 4 \mathrm{ha}$ ) y mayor cantidad de prácticas agrícolas ( $\bar{X}=7.9$ ha) (Candelaria, 20II). El Consejo Nacional de Población (Conapo, 2005) clasificó a cuatro de las seis localidades presentes en la microcuenca (Acazónica, Paso Panal, Patancán y Rancho Nuevo) con alto grado de marginalidad, y a otras dos (Angostillo y Xocotitla) con grado de marginalidad media.

Con base en lo descrito, se planteó como objetivo desarrollar un índice agregado para evaluar la sustentabilidad en los tres grupos de agroecosistemas presentes en la microcuenca Paso de Ovejas i, y se planteó como hipótesis que los sistemas con presencia del componente ganadero presentarían un mayor grado de sustentabilidad debido a un uso complementario de los recursos. 


\section{Metodología}

La MPOV-I, se localiza entre las coordenadas $19^{\circ} 10^{\prime} \mathrm{N}$ y $96^{\circ} 36^{\prime} \mathrm{O} ; 19^{\circ} 13^{\prime} \mathrm{N}$ y $96^{\circ} 35^{\prime} \mathrm{O}$; $19^{\circ} 08^{\prime} \mathrm{N}$ y $96^{\circ} 29^{\prime} \mathrm{O}$; $19^{\circ} 14^{\prime} \mathrm{N}$ y $96^{\circ} 3 \mathrm{I}^{\prime}$ O; tiene una extensión de 7009 has y está formada por las localidades de Acazónica, Angostillo, Paso Panal, Patancán, Rancho Nuevo y Xocotitla (Firco, 2005b). Alberga una población de 256r habitantes y $85 \%$ de la población económicamente activa (PEA) se dedica a las actividades agrícolas (Instituto Nacional de Estadística Geografía e Información [Inegi], 20II). La evaluación de campo se desarrolló en el ciclo agrícola comprendido entre junio de 2009 y enero de 2010 , cuyas precipitaciones correspondieron a un año típico, con un total de $996 \mathrm{~mm}$, 96\% de las cuales se concentró en cinco meses.

Para el presente estudio, se definió la sustentabilidad agrícola como el proceso de obtención de productos agropecuarios que satisfagan necesidades de la población, mediante un conjunto de prácticas agrícolas que logran conservar y mejorar la cantidad y calidad de los recursos naturales, y de este modo asegurar su permanencia y mejorar el desempeño de los AES en el tiempo. Además, estas prácticas favorecen el desarrollo económico, humano y social de las familias que las realizan, lo que contribuye al mejoramiento del entorno.

En esta investigación, primero se establecieron las dimensiones, indicadores $\mathrm{y}$ valores de sustentabilidad acordes al nivel jerárquico, y que fueron definidos y ponderados con base en una consulta tanto a expertos que conocen el área de estudio, como a otros que no conocen el área pero cuentan con experiencia en evaluación de sustentabilidad (Cuadro I). Posteriormente, se entrevistaron 59 de los 65 responsables de AES, con quienes anteriormente se había realizado la caracterización en las seis localidades de la MPOV-I, según Candelaria (20II)². Se utilizó un cuestionario semiestructurado de 36 preguntas, del cual se obtuvieron los valores umbrales para cada indicador. En la valoración de los indicadores, a la mejor opción se le otorgó el valor de uno (I) y a la peor le correspondió cero (o), por lo que no se obtuvieron valores negativos. Estos valores se usaron para la evaluación de la sustentabilidad por localidad y por grupos.

De cada uno de los tres grupos de agroecosistemas resultantes del análisis de conglomerados hechos previamente por Candelaria (20II), se seleccionó al azar un AES que representaba las características del resto de sistemas, por lo que se le

2 Seis productores decidieron no participar. 
denominó agroecosistema Tipo ( $\mathrm{T}_{1}, \mathrm{~T}_{2}$ y $\mathrm{T}_{3}$ ). Al responsable de los AES Tipo, se le aplicó una segunda entrevista de tipo "en profundidad" (Romo y Castillo, 2002; Gallardo, Martínez y Nava , 2010); y para generar información complementaria sobre la dimensión ambiental en cada uno de los tres AES tipo se evaluaron los aspectos físico-biológicos siguientes: cobertura vegetal de los potreros (observación directa); disponibilidad de forraje (con el método del cuadrado medio); erosión de suelo por el método de clavos y rondanas modificado por Mendoza (2005); determinación de la inclinación del terreno (clinómetro electrónico, Mod. 2654I, marca Haglöf Sweden); textura de suelos (Bouyoucos, 1936); y el contenido de N total (microkjeldahl), P (Bray y Kurtz, I945), K (Richter, Conti y Maccarini, 1982) y MO en suelo (Nelson y Sommers, 1986). Para realizar los análisis de suelo se recolectaron muestras aleatorias a una profundidad de $\circ$ a $15 \mathrm{~cm}$ en siete parcelas de los tres AES tipo y bajo diferente uso agrícola.

Para obtener el valor de cada una de las dimensiones se sumaron los valores obtenidos en cada indicador. Luego, para conocer el Índice Agregado de Sustentabilidad Agrícola (IASA), se sumaron los valores de cada una de las cuatro dimensiones, como se representa en la siguiente función:

\section{IASA $=$ ISA+ISE+ISS+ISH}

Donde:

IASA= Índice Agregado de Sustentabilidad Agrícola (valor mínimo o y máximo I). ISA= Índice de Sustentabilidad Ambiental (valor mínimo o y máximo o.3).

ISE= Índice de Sustentabilidad Económica (valor mínimo o y máximo 0.25).

ISS= Índice de Sustentabilidad Social (valor mínimo o y máximo 0.15).

ISH= Índice de Sustentabilidad Humana (valor mínimo o y máximo 0.3).

Para determinar la sustentabilidad de los AES de la microcuenca MPOV-i y comparar entre localidades y entre grupos, se utilizó el programa Excel Office 2007, para el análisis estadístico del Índice Agregado de Sustentabilidad se usó el paquete Statisticsv6 $6^{3}$ y también se realizó un análisis de varianza y prueba de medias Tukey $(\mathrm{P} \leq 0.05)$.

3 Statistica V6. 2002. StatSoft, Inc. 2300, Computer Program Manual. Tulsa. 
Cuadro 1. Dimensiones, indicadores y valores para determinar la sustentabilidad de los agroecosistemas de la microcuenca Paso de Ovejas I
Dimensión
Clave Indicador
VALORACIÓN

Ambiental

DC Diversidad de Cultivos +2cultivos $=0.03$; $2 \mathrm{C}=0.02$; IC $=0.01$ y $\circ \mathrm{C}=0$

\begin{tabular}{cl}
\hline DG Diversidad Ganadera & $\begin{array}{l}+2 \text { especies de ganado }=0.03 ; \\
\text { 2eg }=0.02 ; \text { reg }=0.01 \text { y } \circ \text { eg }=0\end{array}$ \\
\hline
\end{tabular}

CS Conservación de Suelo 2prácticas $=0.04 ;$ ip $=0.02$ y op=0

CA Conservación de Agua 2prácticas $=0.04 ; \mathrm{rp}=0.02$ y $\circ \mathrm{p}=0$

\begin{tabular}{|c|c|c|}
\hline $\mathrm{TF}$ & Tipo de Fertilización & $\begin{array}{l}\text { Mixta }=0.03 ; \text { Orgánica }=0.02, \\
\text { Sintética }=0.01 \text { y Ninguna=0 }\end{array}$ \\
\hline UP & $\begin{array}{l}\text { Uso de Agroquímicos } \\
\text { en Cultivos }\end{array}$ & Bajo=0.03; Medio=0.02; Alto=0.01 y Ninguno=0 \\
\hline UAG & $\begin{array}{l}\text { Uso de Agroquímicos } \\
\text { en Ganado }\end{array}$ & Bajo $=0.02 ;$ Medio $=0.01$ y Alto $=0$ \\
\hline $\mathrm{TP}$ & Tipo de Potrero & $\begin{array}{l}\text { Silvopastoreo=0.03; Pasto Mejorado=0.02; } \\
\text { Grama Nativa }=0.01 \text { y Sin Cobertura Herbácea }=0\end{array}$ \\
\hline $\mathrm{ES}^{*_{1}}$ & Erosión de Suelo & $\begin{array}{l}\text { Sin Erosión }=0.04 \text {; Erosión Laminar }=0.03 \text {; } \\
\text { Escalones Incipientes }=0.02 \text {; Escalones } \\
\text { Consolidados }=0.01 \text {; Cárcavas }=0\end{array}$ \\
\hline NIA & $\begin{array}{l}\text { Nivel de Ingresos } \\
\text { por Agricultura }\end{array}$ & $\begin{array}{l}+2 \text { salarios mínimos=0.03; } \\
2 \mathrm{sm}=0.02 ; \mathrm{Ism}=0.01 \text { y }-\mathrm{Ism}=0\end{array}$ \\
\hline EIA & $\begin{array}{l}\text { Estacionalidad del } \\
\text { Ingreso Agrícola }\end{array}$ & $\begin{array}{l}+8 \mathrm{meses}=0.04 ; \text { De } 6 \mathrm{a} 8 \mathrm{~m}=0.03 ; \text { De } \\
4 \mathrm{a} 5 \mathrm{~m}=0.02 ; \text { De } 2 \mathrm{a} 3 \mathrm{~m}=0.01 ; \mathrm{Im}=0\end{array}$ \\
\hline $\mathrm{EE}$ & Empleo Extrafinca & $\begin{array}{l}\text { Sin Ingresos Extrafinca=0.04; Percibe Uno } \\
\text { (divisas o extrafinca) }=0.02 \text { y Percibe Ambos }=0\end{array}$ \\
\hline $\mathrm{SA}^{* 2}$ & Seguridad Alimentaria & De $5 \mathrm{a} 6=0.06 ;$ De $4 \mathrm{aj}=0.04 ;$ De $2 \mathrm{a} \mathrm{I}=0.02 \mathrm{y}<\mathrm{I}=0$ \\
\hline
\end{tabular}

PC Pérdidas en Cultivos De 5 a $15 \%=0.02$; De 15 a $25 \%=0.01$ y $>25 \%=0$

PG Pérdidas en Ganadería De 5 a $15 \%=0.02$; De 15 a $25 \%=0.01$ y $>25 \%=0$

RP Renta de Pasto $\quad$ meses=0.04; De razm=0.03; de

$4 \mathrm{a} 6 \mathrm{~m}=0.02$; De 7 a9m=0.01 $\mathrm{y}>\mathrm{IOm}=0$ 
Social

$\mathrm{CC}^{*} 3$ Cursos de Capacitación $\quad+2$ cursos $=0.03 ; 2 \mathrm{C}=0.02$, IC $=0.01$ y $\circ \mathrm{C}=0$

AC Acceso a Créditos Sí=o.or y No=o

\begin{tabular}{cll} 
AS & $\begin{array}{l}\text { Acceso a Programas } \\
\text { de Asistencia Social }\end{array}$ & Ningún Programa $=0.03 ;$ ip $=0.02 ; 2 \mathrm{p}=0.01 \mathrm{y}+2 \mathrm{p}=0$ \\
\hline OP & $\begin{array}{l}\text { Organización con } \\
\text { Productores }\end{array}$ & $\begin{array}{l}\text { Sí=0.04; Perteneció a una } \\
\text { organización }=0.02 \text { y no=0 }\end{array}$ \\
\hline VP & $\begin{array}{l}\text { Comercialización de } \\
\text { Productos Agrícolas }\end{array}$ & $\begin{array}{l}\text { Consumidor }=0.03 ; \text { Intermediario }=0.02 \\
\text { y Ninguno }=0\end{array}$ \\
\hline
\end{tabular}

Humano

ER Edad del Responsable

De 30-40 años=0.05; De 4I-50=0.04; De 5I-

$60=0.03$; De $61-70=0.02 ;$ De $71-80=0.01$ y $>80=0$

\begin{tabular}{|c|c|c|}
\hline EsR & $\begin{array}{l}\text { Escolaridad del } \\
\text { Responsable }\end{array}$ & De 7 a 9 años=0.02; De i a $6=0.01$ y Ninguno $=0$ \\
\hline EM & $\begin{array}{l}\text { Escolaridad Máxima } \\
\text { en el AES }\end{array}$ & $\begin{array}{l}\text { De Iз а I } 6=0.04 ; \text { De I0 a I } 2=0.03 ; \text { De } 7 \text { a } \\
9=0.02 ; \text { De г a } 6=0.01 \text { y Ninguno=0 }\end{array}$ \\
\hline GS & $\begin{array}{l}\text { Satisfacción por } \\
\text { la Agricultura }\end{array}$ & $S_{1}^{\prime}=0.03$ y $N_{0}=0$ \\
\hline DCP & $\begin{array}{l}\text { Deseos del Productor de } \\
\text { Continuar en Agricultura }\end{array}$ & $\mathrm{S}_{1}^{\prime}=0.03$ y No=0 \\
\hline $\mathrm{DCH}$ & $\begin{array}{l}\text { Deseos del Heredero } \\
\text { de Continuar en } \\
\text { la Agricultura }\end{array}$ & $S_{1}^{\prime}=0.05$ y No=0 \\
\hline SB & Acceso a Servicios Básicos & $\begin{array}{l}4 \text { servicios }=0.04 ; 3 \mathrm{~s}=0.03 ; 2 \mathrm{~S}=0.02 \\
\text { IS }=0.0 \text { I y Ninguno }=0\end{array}$ \\
\hline EG & $\begin{array}{l}\text { Presencia de Enfermedad } \\
\text { Grave en la Familia }\end{array}$ & $\mathrm{N}_{0}=0.04$ y $\mathrm{Sí}_{1}=0$ \\
\hline
\end{tabular}

* Factor de corrección:

${ }^{\mathrm{I}}$ Erosión activa (-०.০I), Erosión Estabilizada (০) y Recuperación de Suelo (+०.০I); ${ }^{2}$ Relación: Miembros Productores/Miembros Consumidores (R:P/C). R:P/CDo.3 (-0.0I), R:P/C de 0.3 a 0.6 (o) y R:P/C<0.6 (+o.oI); ${ }^{3}$ Familiares que asistieron al curso.

Familiar que no toma decisiones (-0.0I),

Responsable solo (०) y Responsable + familiar joven (+०.0I)

Fuente: elaboración propia 


\section{Resultados y discusión}

El grado de sustentabilidad de los AES de la MPOV-I en general fue bajo (0.52).

Entre localidades no hubo diferencias estadísticas ( $\mathrm{P}>0.05)$, lo que sí se observó entre grupos $(\mathrm{P}<0.05)$, donde $\mathrm{G}$ r registró mayor grado de sustentabilidad (0.58), seguido de $\mathrm{G}_{3}$ y $\mathrm{G}_{2}$ con 0.53 y 0.48 , respectivamente (Cuadro 2). La diferencia se debe a que los productores del Gi poseen mayor disponibilidad de tierras, por lo que logran integrar con mayor eficiencia el componente animal y los cultivos. Usan el rastrojo de maíz para alimentar al ganado en la época seca, y el aporte de estiércol por parte de ganado durante el pastoreo. Estas son las interacciones más importantes en las condiciones de la MPOV-I que, por el contrario, están ausentes en los AES con menor disponibilidad de tierras.

Se observó un comportamiento similar en las cuatro dimensiones del Índice Agregado de Sustentabilidad Agrícola (IASA) en las seis localidades. El eje con menor valor y mayor similitud entre localidades fue el social ( 0.3 a 0.4 ), debido a que en la MPOV-I se ha reproducido el mismo patrón de comportamiento social. Este patrón se caracteriza por una fuerte indiferencia con respecto a la organización, alta dependencia de programas asistenciales gubernamentales y emigración. La emigración es un problema que ha sido documentado en la microcuenca, y son varios los destinos elegidos (tanto estatales como nacionales o internacionales). Según Gallardo et ál. (2010), Acazónica es la localidad con mayor emigración, pues $25 \%$ de su población se encuentra en Estados Unidos.

CuAdro 2. Índice Agregado de Sustentabilidad de los agroecosistemas por localidad y grupo en la microcuenca Paso De Ovejas I

\begin{tabular}{|c|c|c|c|c|}
\hline LOCALIDAD & $\mathrm{N}$ & Mínimo & Máximo & Promedio \\
\hline Acazónica & 19 & 0.29 & 0.63 & 0.53 \\
\hline Angostillo & 9 & 0.33 & 0.63 & 0.48 \\
\hline Paso Panal & 4 & 0.42 & 0.57 & 0.50 \\
\hline Patancán & 10 & 0.36 & $0.6 \mathrm{I}$ & 0.48 \\
\hline Rancho Nuevo & 5 & 0.42 & 0.65 & 0.54 \\
\hline Xocotitla & 9 & 0.49 & 0.60 & 0.55 \\
\hline \multicolumn{5}{|l|}{ GRUPo } \\
\hline I & IO & 0.51 & 0.68 & $0.58 \underline{a}$ \\
\hline 2 & 23 & 0.33 & 0.64 & $0.48^{\mathrm{b}}$ \\
\hline 3 & 23 & $0.4 \mathrm{I}$ & 0.63 & $0.53^{\mathrm{b}}$ \\
\hline
\end{tabular}


El efecto de las remesas se manifestó en las dimensiones económica y humana. En la primera, se dio un valor bajo de sustentabilidad cuando se presentó ingreso por trabajo extrafinca o remesas, y un valor alto cuando no se registró tal ingreso; sin embargo, fue evidente que las familias con acceso a remesas satisfacen mejor sus necesidades de alimentación, educación, salud, esparcimiento y casa habitación. La dimensión humana presentó la mayor variación entre localidades: fue mayor en Acazónica (0.74) y menor en Paso Panal (0.5), que resultaron además las localidades con el mayor y menor índice de emigración respectivamente.

En la Figura i se muestran los 29 indicadores por separado. Allí se observa una baja diversidad de cultivos (DC) y ganadera (DG), bajo número de prácticas de conservación de agua (PCA) y suelo (PCS) (I.5 en promedio por cada una), pese a que existe conocimiento y preocupación de los productores con respecto a la pérdida de suelo y agotamiento de las fuentes de almacenamiento de agua. El indicador utilizado del tipo de fertilizante (TF) mostró valor elevado, debido a que en la mayoría de los casos existe fertilización con productos sintéticos y aporte de excretas del ganado bovino que pastorea el rastrojo en las áreas de cultivo.

Para el uso de los indicadores de agroquímicos se otorgó mayor valor a quienes emplean menor cantidad de estos en sus prácticas, y menor valor a quienes emplean mayor cantidad. El indicador de uso de agroquímicos en cultivos (PC) fue bajo (0.2) debido a la alta aplicación de estos en cultivos para el control de plagas y arvenses, mientras que el uso de agroquímicos en ganadería (UPG) fue alto (o.9), debido a que los AES con tendencia a la ganadería emplean menos agroquímicos en su manejo, el cual es extensivo y rudimentario. Sin embargo, se observó poco interés en mejorar las condiciones de la ganadería, debido a que la baja rentabilidad y pérdidas son menos evidentes en el corto tiempo en comparación con las mermas que se presentan en los ciclos de cultivos; incluso, cuando no se obtienen cosechas de maíz, se dispone de su forraje para el ganado, lo que da al productor una impresión de rentabilidad ganadera.

Los potreros se encuentran constituidos principalmente por una mezcla de gramíneas nativas y mejoradas, con bajo nivel productivo debido a que se localizan en suelos erosionados; prácticas de fertilización inexistentes; manejo inadecuado de la carga animal (sobrepastoreo); y un periodo aproximado de ocho meses de escasez de precipitaciones. Como respuesta a esta situación los AES de Gi y G3, con tendencia a la ganadería, han adoptado de forma incipiente la práctica de sistemas silvopastoriles y, por lo general, se valen del pasto guinea (Panicum maximum) con guácimo (Guazuma ulmifolia), así como la elaboración de silos de fermentación de maíz. 

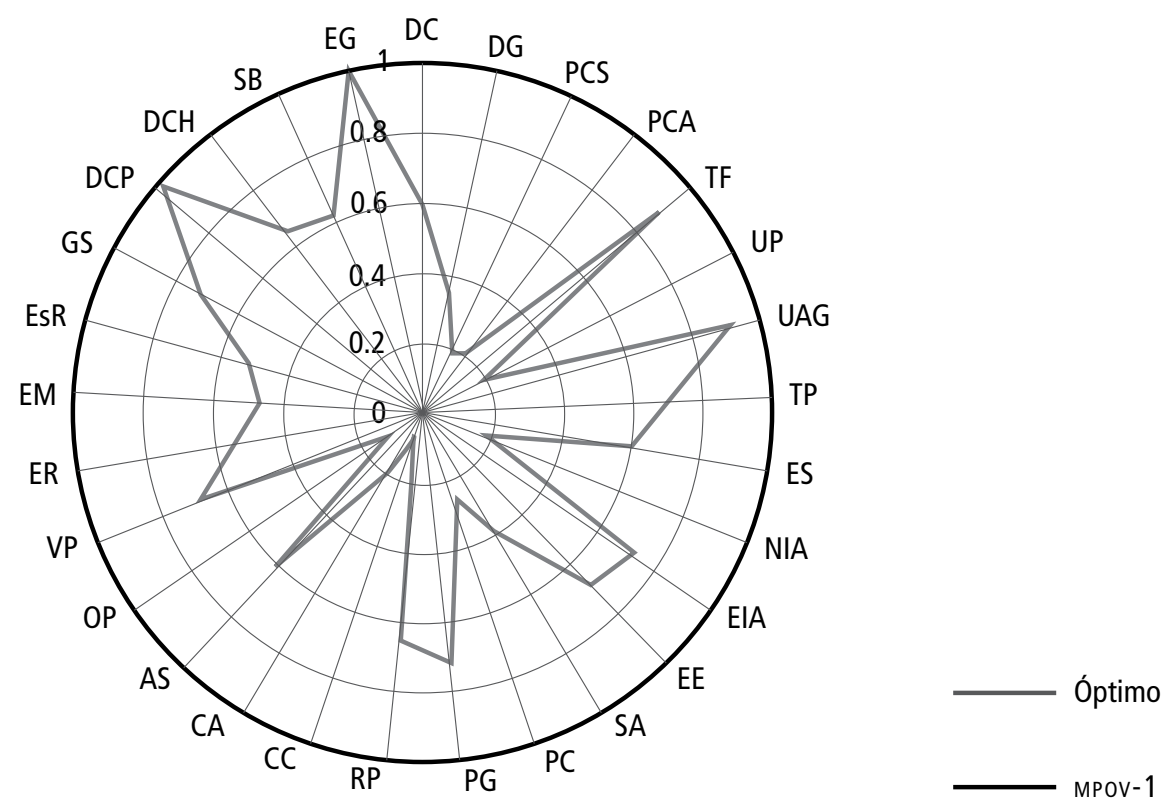

Figura i. Indicadores de sustentabilidad de los agroecosistemas de la microcuenca Paso de Ovejas i, municipio de Paso de Ovejas, Veracruz

Fuente: elaboración propia

Cuando se evaluó el ingreso económico de los AES de la MPOV-i producto de la actividad agrícola, calculado en salarios mínimos (sm), donde un salario mínimo de la zona equivale a US $\$ 3.8^{4}$; se encontró que en general fue bajo, en todos los casos menor a 2.5, lo que representó un valor del indicador del nivel de ingresos por agricultura (NIA) de 0.19 (Figura I); los valores más altos se observaron en Xocotitla ( $\bar{X}=1.2 \mathrm{sm})$, y los más bajos en Rancho Nuevo ( $\bar{X}=0.5 \mathrm{sm})$.

Esta situación ha originado que los integrantes de las familias agrícolas recurran al empleo extrafinca para cubrir sus necesidades económicas, lo que implica desempeñarse como jornaleros agrícolas, albañiles en actividades de comercio en la región, además de la migración a Estados Unidos. Esta situación origina un proceso de desterritorialización que, según Entrena (1999), "es un éxodo poblacional del campo que provoca el descontrol de los procesos de acción productiva, organizativa, relacional y cultural, en la región”. En algunos casos, cuando se presentaron pérdidas económicas en la actividad ganadera o en cultivos, los ingresos por el trabajo extrafinca o por divisas atenuaron u ocultaron el problema.

4 El salario mínimo es definido anualmente por mandato constitucional por la Comisión Nacional de los Salarios Mínimos, es el ingreso diario en pesos mexicanos. 
En el periodo de estudio, las pérdidas fueron mayores en cultivos que en ganado; en algunos casos de cultivo de maíz llegó a 100\%, y hasta $15 \%$ en el ganado. Sin embargo, el siniestro de una hectárea de maíz en etapa reproductiva, representó una disponibilidad de 3.3 t MS de rastrojo útil para el ganado en el AES T2. La seguridad alimentaria en los AES evaluados fue baja, debido en general al abandono del manejo de los traspatios, que representaba una fuente importante de alimentos para la familia, al ofrecer proteína de origen animal y hortalizas. El análisis mostró que 56\%, 61\% y 39\% de los productos básicos (maíz, frijol, chile, leche, huevo, carne de pollo y frutas varias) fueron adquiridos en el mercado en AES TI, $T_{2}$ y $T_{3}$, respectivamente.

Los cursos de capacitación ofertados han sido pocos en los últimos cuatro años, salvo los promovidos por investigadores del Colegio de Postgraduados-Veracruz sobre sistemas silvopastoriles, y dos más abiertos por el Gobierno Municipal sobre elaboración de escobas artesanales y cría de cerdos. Por otro lado, la edad de los productores es un factor limitante para la implementación de prácticas de manejo consideradas como sustentables: el promedio es de 57 años, con un mínimo de 24 y un máximo de 87; además, $15 \%$ tiene más de 70 años de edad.

También se observó que, en algunos casos, los herederos de los AES no desean continuar con la actividad agrícola, por lo que se pronostica que a mediano plazo se incrementará el abandono de los sistemas de producción agrarios en la MPOV-I, y las familias se verán en la necesidad de adoptar nuevas formas de empleo o migrar hacia los polos de desarrollo. Por otra parte, la presencia de enfermedades graves en la familia es un fenómeno relevante que afectó la sustentabilidad de los AES, en especial, por los gastos económicos generados por servicios médicos, y porque implicó que algún familiar sano dedicara tiempo para atender al familiar enfermo. Esto disminuyó la capacidad de trabajo de la familia, principal fuente de mano de obra.

Los resultados del análisis de suelo de los AES Tipo muestran un bajo contenido de nutrientes en todos los casos, y extremos en algunos (Cuadro 3); se encontró que el único elemento utilizado de forma regular por los productores para fertilizar el cultivo del maíz, es $\mathrm{N}$ en forma de úrea, y esporádicamente $\mathrm{K}$ y $\mathrm{P}$, en bajas dosis. Por tanto, es necesario establecer estrategias para mejorar el contenido de $\mathrm{MO}$, por ser un factor clave en el proceso de recuperación química y física del suelo (Morón, 2003; García, 2006; Janzen, 2006; Osuna, Figueroa, Oleschko, Flores, Martínez y González, 2006). Esto podría realizarse con la implementación de tecnologías agroecológicas, como el uso de abonos orgánicos 
Cuadro 3. Características físicas y químicas del suelo de los agroecosistemas tipo de la microcuenca Paso De Ovejas I

\begin{tabular}{lllllll}
\hline Tipo & Cultivo & Textura & M.O. (\%) & N (РPм) & $\begin{array}{l}\text { PO } 4 \\
\text { (РPм) }\end{array}$ & K (РPм) \\
\hline I & Maíz & Migajón arcilloso & 2.3 & 0.10 & 0 & 20 \\
\hline I & Potrero & Migajón arcilloso & 3 & 0.15 & 0.5 & 20 \\
\hline 2 & Maíz & Franco & 2.09 & 0.10 & I & 20 \\
\hline 2 & Potrero & Migajón arenoso & I.2 & 0.06 & I & 25 \\
\hline 3 & Maíz & Migajón arcilloso & 2.2 & 0.10 & 0 & 25 \\
\hline 3 & Maíz & Franco & 3 & 0.50 & I & \multirow{2}{*}{10} \\
\hline 3 & Potrero & Migajón arcilloso & 3.5 & 0.17 & 0 & 25 \\
\hline
\end{tabular}

FuENTE: elaboración propia

en sus distintas modalidades, como lo recomiendan Sánchez-Hernández, Ordaz-Chaparro, Benedicto-Valdés, Hidalgo-Moreno y Palma-López (2006); y Viteri, Martínez y Bermúdez (2008); con el establecimiento de sistemas agro y silvopastoriles (que tienen la capacidad de reciclar nutrientes y evitar la erosión y degradación del suelo); incrementar la calidad de la dieta animal (López-Ortiz, Pérez-Hernández, Ahuja-Aguirre, Ortega-Jiménez, et ál., 2009); y cuidar la fauna edáfica, mediante la disminución del uso de agroquímicos, (por su nicho ecológico presentan una relación directa con el equilibrio de la dinámica de los ecosistemas), según Flota-Bañuelos et ál., 20ı2, y García, 20 I.

Durante el periodo de estudio se presentó un proceso de erosión hídrica del suelo (Cuadro 4), ubicado entre parámetros de normal a moderada según la clasificación de la FAO de 1980 , descrita por Mendoza (2005). Se registró una menor pérdida de suelos con establecimiento de pasto, en comparación con suelos utilizados para el cultivo de maíz, debido a que estos últimos se aran cada dos años, o incluso cada año, según el criterio del productor o la disponibilidad de recursos económicos. Por otra parte, los suelos destinados al cultivo de maíz se localizan en laderas con pendientes pronunciadas, factor que, aunado a la práctica del arado del suelo, presencia de precipitaciones concentradas en pocos días del periodo de lluvias y la falta de cobertura del vegetal, propicia que los índices de erosión se incrementen. 
Cuadro 4. Erosión hídrica de suelo de los agroecosistemas tipo de la microcuenca Paso De Ovejas I

\begin{tabular}{|c|c|c|c|c|}
\hline Tipo & Uso AGRÍCOLA & Pendiente (\%) & EROSIón (T HA ${ }^{-1}$ AÑO $\left.{ }^{-1}\right)$ & INTENSIDAD \\
\hline I & Maíz & 7 & I.5 & Ligera \\
\hline I & Potrero & 19 & $0.3^{\underline{a}}$ & Normal \\
\hline 2 & Maíz & 13 & $2.3^{\mathrm{ab}}$ & Ligera \\
\hline 2 & Maíz & 13 & $0.9^{\underline{\mathrm{a}}}$ & Ligera \\
\hline 3 & Maíz & 9 & $1.5^{\mathrm{ab}}$ & Ligera \\
\hline 3 & Maíz & 24 & $7.6^{\mathrm{b}}$ & Moderada \\
\hline 3 & Potrero & 5 & $0.5^{\underline{\mathrm{a}}}$ & Normal \\
\hline
\end{tabular}

FuENTE: elaboración propia

\section{Conclusiones}

Se concluye que en la microcuenca Paso de Ovejas i se realiza un manejo deficiente de los recursos naturales y productivos, esto es, que no se aprovecha el potencial de la región, lo que origina un bajo grado de sustentabilidad. Se observaron restricciones en el ámbito ambiental, social y económico para el desenvolvimiento de los agroecosistemas, lo cual afecta el desarrollo humano de las familias agrícolas.

Se considera que la dimensión social es prioritaria en el desarrollo de alternativas de sustentabilidad, con particular importancia en el proceso de organización de los productores como mecanismo para innovar los sistemas de producción, mediante la adopción, implementación y adecuación de tecnologías generadas en el enfoque agroecológico. Es necesario recuperar el manejo de los huertos de traspatios como estrategia para alcanzar la seguridad alimentaria y la revaloración cultural entre las generaciones jóvenes, así como proceso de educación ambiental en la categoría de no formal.

En la investigación fue posible obtener un índice agregado de la sustentabilidad de los agroecosistemas de la microcuenca Paso de Ovejas i. Los sistemas con tendencia a la ganadería presentaron mayor grado de sustentabilidad, debido a que hacen un uso más integral de los recursos naturales y productivos, así como un menor uso de insumos, sobre todo agroquímicos y mayor ciclaje de nutrientes. 


\section{Agradecimientos}

Los autores agradecen a los productores de la microcuenca Paso de Ovejas i por la información brindada; al Consejo Nacional de Ciencia y Tecnología (Conacyt) por la beca de doctorado otorgada a Bernardino Candelaria Martínez. A la Línea Prioritaria de Investigación en Agroecosistemas Sustentables del Colegio de Postgraduados y al Colegio de Postgraduados, Campus Veracruz, por el financiamiento de la investigación y las facilidades para su desarrollo. Asimismo, agradecen los comentarios de los revisores anónimos, gracias a los cuales se mejoró sustancialmente la calidad del escrito.

\section{Referencias}

Altieri, M. A. y Nicholls, C. (2012). Agroecología: única esperanza para la soberanía alimentaria y la resiliencia socioecológica. Sociedad Científica Latinoamericana de Agroecología (Socla). Recuperado el 25 de junio de 20I2, de http://riozo.net/ wp-content/uploads/2012/06/final2.pdf

Bartlett, A. A. (2006). Reflexiones sobre sostenibilidad, crecimiento de la población y medio ambiente. Population \& Environment, I6(I), 5-35.

Belcher, K. W., Boehm, M. M. y Fulton, M. E. (2004). Agroecosystem Sustainability: a System Simulation Model Approach. Agricultural Systems, 79(2), 225-24I.

Bouyoucos, G. S. (1936). Directions for Making Mechanical Analysis of Soil by Hydrometer Method. Soil Science, 42(3), 225-229.

Bray, R. y Kurtz, L. (1945). Determination of Total, Organic and Available Forms of Phosphorus in Soil. Soil Science, 59(I), 39-45.

Cáceres, D. (2004). Sostenibilidad como concepto situado. Un marco conceptual para la construcción de indicadores. Desarrollo rural y cooperativismo agrario, (8), I89-200.

Cáceres, D. (2009). La sostenibilidad de explotaciones campesinas situadas en una reserva natural de Argentina central. Agrociencia, 43(5), 539-550.

Candelaria, M. B. (20II). Diseño participativo para mejorar la sustentabilidad de los agroecosistemas de la microcuenca Paso de Ovejas i en el estado de Veracruz, México. (Tesis para optar al grado de Doctor en Ciencias, publicada) Colegio de Postgraduados, Veracruz, México.

Candelaria, M. B., Ruiz, R. O., Gallardo, L. F., Pérez, H. P., Martínez, B. A. y Vargas, V. L. (20II). Aplicación de modelos de simulación en el estudio y 
planificación de la agricultura, una revisión. Tropical and Subtropical Agroecosystem, I4(3), 999-1010.

Colás, B. P. (200I). Educación e investigación en la sociedad del conocimiento: enfoques emergentes. Revista de Investigación Educativa, 19(2), 291-313.

Consejo Nacional de Población [Conapo] (2005). Indice de marginación a nivel localidad. Recuperado el 2 de diciembre de 2010, de http://www.conapo.gob.mx/es/ CONAPO/Indice_de_Marginacion_por_Localidad_2010

Consejo Nacional de Evaluación de la Política de Desarrollo Social [Coneval] (2007). Mapas de pobreza por ingresos y rezago social. Recuperado el 20 de octubre de 20II, de http://www.coneval.gob.mx/rw/resource/coneval/med/pobreza/ Veracruz_de_Ignacio_de_la_Llave_05.pdf

Entrena, D. F. (1999). La desterritorialización de las comunidades locales rurales y su creciente consideración como unidades de desarrollo. Revista de Cooperativismo Agrario y Desarrollo Rural, (3), 29-4I.

Fideicomiso de Riesgo Compartido [Firco] (2005a). Guía técnica para la elaboración de planes rectores de producción y conservación (PRPC). México D.F.: Secretaría de Agricultura, Ganadería, Desarrollo Rural, Pesca y Alimentación.

Firco (2005b). Plan rector de producción y conservación Paso de Ovejas I. Paso de Ovejas, Veracruz: Secretaría de Agricultura, Desarrollo Rural, Pesca y Alimentación. Flota-Bañuelos, C., Lopéz-Collado, J., Vargas-Mendoza, M., Fajersson, P., GonzálezHernández, H. y Martínez-Morales, I. (20I2). Efecto de la ivermectina en la dinámica espacio-temporal de escarabajos estercoleros en Veracruz, México. Tropical and Subtropical Agroecosystem, ${ }_{15}$ (2), 227-239.

Gallardo, L. F., Martínez, G. S. E. y Nava, T. M. E. (2010). Redes migratorias y procesos de toma de decisiones en los agroecosistemas de Acazónica y Hato de la Higuera, Veracruz. En T. Nava (Ed.), Migración y desarrollo rural en cuatro regiones campesinas de Veracruz (pp.ı03-I24). México, D. F: Colegio de Postgraduados, Universidad Autónoma Chapingo.

García, F. (2006). La nutrición de los cultivos y la nutrición de los suelos. Informaciones Agronómicas del Cono Sur, (29), I3-16.

García, de S. I. E. (20II). Microorganismos del suelo y sustentabilidad de los agroecosistemas. Revista Argentina de Microbiología, 43(I), I-3.

Instituto Mexicano para la Competitividad A.C [IMCO] (2007). Situación de la competitividad de México-2006: punto de inflexión. Recuperado el I2 de marzo de 20II, de imco.org.mx/wp-content/uploads/2006/ı2/._de_inflexion_situacion_de_la_ competitividad_de_mexico_2006.pdf 
Instituto Nacional de Estadística Geografía e Información [Inegi] (20II). Censo nacional de población y vivienda. Recuperado el I2 de marzo de 20II, de http://www. inegi.org.mx/sistemas/olap/proyectos/bd/consulta.asp?p=I7II \&\&c=27769\&s=est\# Janzen, H. H. (2006). The Soil Carbon Dilemma: Shall We Hoard it or Use it?. Soil Biology and Biochemistry, 38(3), 419-424.

López-Ortiz, S., Pérez-Hernández, P., Ahuja-Aguirre, C. C., Ortega-Jiménez, E., Díaz-Rivera, P., Olguín-Palacios, C., Rosendo-Ponce, A., Gallardo-López, F. y Gallegos-Sánchez, J. (2009). En S. Tejeda, O. Suarez, M. Pro, A. Torres y S. Gallegos (Eds.), Curso de reproducción en rumiantes: nuevas alternativas de manejo para mejorar la eficiencia reproductiva (pp. 4I-50). Montecillo: Colegio de Postgraduados.

Masera, O., Astier, M. y López, R. S. (1999). Sustentabilidad y manejo de recursos naturales (el marco de evaluación MESMIS). México, D. F.: GIRA, UNAM.

McMichael, A. J., Butler, C. D. y Folke, C. 2003. New Visions for Addressing Sustainability. Science, 302(5652), I919-1920

Mendoza, A. M. (2005). Manual de métodos sencillos para estimar erosión hídrica. Documento 502, serie técnica 5/2005. Managua: UNA, Pasolac, CIAT.

Morón, A. (2003). Principales contribuciones del experimento de rotaciones cultivospasturas del INIA La Estanzuela en el área de fertilidad de suelos (I963-2003). En Simposio 40 años de rotaciones agrícola-ganaderas. Serie Técnica 134. La Estanzuela: Autor. Recuperado el 24 de octubre de 20I0, de http://www.ipni.net/ppiweb/

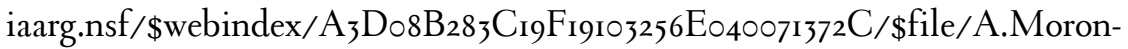
Rotaciones-INIA+La+Estanzuela.pdf

Nahed, T. J., Castel, J. M., Mena, Y. y Caravaca, F. (2006). Appraisal of the Sustainability of Dairy Goat Systems in Southern Spain According to their Degree of Intensification. Livestock Science, IOI(I-3), I0-23.

Nelson, D. W. y Sommers, L. E. (1986). Total Carbon, Organic Carbon and Organic Matter. En A. Klute (Ed.), Methods of Soil Analysis. Part 2. Chemical and Microbiological Proprieties (pp. 539-547). Wisconsin: Madison.

Osuna, C. E., Figueroa, S. B., Oleschko, K., Flores, D., Martínez, M. M. y González, C.F. (2006). Efectos de la estructura del suelo sobre el desarrollo radical del maíz con dos sistemas de labranza. Agrociencia, 40(I), 27-38.

Richter, M., Conti, M. E. y Maccarini, G. (1982). Mejoras en la determinación de cationes intercambiables, acidez intercambiable y capacidad de intercambio catiónica. Revista de la Facultad de Agronomía, (3), I45-I47.

Romo, M. M. y Castillo, D. C. (2002). Metodologías de las ciencias sociales aplicadas al estudio de la nutrición. Revista Chilena de Nutrición, 29(I), I4-22. 
Ruiz, R. O. (200I). The Systems Approach for Sustainable Development at Catchment and Parish Group Levels. International Journal of Sustainable Development and World Ecology, 8(I), 79-84.

Sánchez-Hernández, R., Ordaz-Chaparro, V. M., Benedicto-Valdés, G. S., HidalgoMoreno, C. L. y Palma-López, D. J. (2006). Regeneración estructural de un suelo arcilloso por aportes de vermicompost en la Chontalpa, Tabasco, México. Universidad y Ciencia, 22(I),13-26.

Sepúlveda, S., Chavarría, H., Rojas, P., Castro, A., Bolaños, D., Picado, E. y Guilleme, M. (2005). Metodología para estimar el nivel de desarrollo sostenible en territorios rurales (El Biograma). San José: Instituto Interamericano de Cooperación para la Agricultura.

Torres, L. P., Rodríguez, S. L. y Sánchez, J. O. (2004). Evaluación de la sustentabilidad del desarrollo regional. El marco de la agricultura. Región y Sociedad, I6(29), 109-144.

Urzelai, A., Olazábal, M., García, G., Santa Coloma, O., Herranz, K., Abajo, B., Acero, J. A., Feliu, E. y Aspuru, I. (2006). Modelización de un sistema territorial "urbano-rural" para la evaluación de su sostenibilidad. Aplicación a una zona representativa de País Vasco. Sostenibilidad, Tecnología y Humanismo, (I), I59-I72. Vant, A., Bakken, L., Bleken, A. M., Baadshaug, H. O., Fykse, H., Haugen, E. L., Lundekvam, H., Morken, J., Romstad, E., RØrstad, K. P., Skjelvåg, O. A. y Song, T. (2006). A Methodology for Integrated Economic and Environmental Analysis of Pollution from Agriculture. Agricultural Systems, 88(2-3), 270-293. Viteri, S. E., Martínez, J. W. y Bermúdez, A. C. (2008). Selección de abonos verdes para los suelos de Turmequé (Boyacá). Agronomía Colombiana, 26(2), 332-339. 